\title{
Downregulation of microRNA-1274a induces cell apoptosis through regulation of BMPR1B in clear cell renal cell carcinoma
}

\author{
HIROFUMI YOSHINO*, TOMOKAZU YONEZAWA*, MASAYA YONEMORI, \\ KAZUTAKA MIYAMOTO, TAKASHI SAKAGUCHI, SATORU SUGITA, YOUICHI OSAKO, \\ SHUICHI TATARANO, MASAYUKI NAKAGAWA and HIDEKI ENOKIDA \\ Department of Urology, Graduate School of Medical and Dental Sciences, \\ Kagoshima University, Kagoshima 890-8520, Japan
}

Received May 24, 2017; Accepted October 12, 2017

DOI: $10.3892 /$ or.2017.6098

\begin{abstract}
Our previous studies of the microRNA (miRNA) expression signature in clear cell renal cell carcinoma (ccRCC) indicated that miRNA-1274a (miR-1274a) was significantly upregulated in clinical specimens, suggesting that $m i R-1274 a$ may act as an oncogenic miRNA in ccRCC. The aim of this study was to investigate the functional roles of $m i R-1274 a$ and identify downstream tumor-suppressive targets regulated by $m i R-1274 a$ in ccRCC cells. Functional studies of $m i R-1274 a$ were carried out by anti-miRNA to investigate cell proliferation and apoptosis using the A498, ACHN and Cakil ccRCC cell lines. Suppression of $m i R-1274 a$ significantly inhibited cancer cell proliferation and induced apoptosis in the ccRCC cells. Gene expression data combined with in silico analysis and luciferase reporter assays demonstrated that bone morphogenetic protein receptor type $1 \mathrm{~B}$ (BMPR1B) was directly regulated by $m i R-1274 a$. Moreover, TCGA database as well as immunohistochemistry demonstrated low expression of BMPR1B in ccRCC clinical specimens compared to that in normal kidney tissues. We conclude that loss of oncogenic miR-1274a reduced cancer cell proliferation and induced apoptosis in ccRCC through targeting BMPR1B. Our data revealing molecular pathways and a target gene regulated by oncogenic $m i R-1274 a$ provide new insight into the potential mechanisms of ccRCC oncogenesis.
\end{abstract}

\section{Introduction}

Renal cell carcinoma (RCC) is a disease in which cells in the tubules of the kidney undergo oncogenic transformation. The most common subtype (approximately $80 \%$ of cases) of $\mathrm{RCC}$ is clear cell RCC (ccRCC) (1). The 5-year survival rate of

Correspondence to: Dr Hideki Enokida, Department of Urology, Graduate School of Medical and Dental Sciences, Kagoshima University, 8-35-1 Sakuragaoka, Kagoshima 890-8520, Japan

E-mail: enokida@m.kufm.kagoshima-u.ac.jp

${ }^{*}$ Contributed equally

Key words: microRNA, miR-1274a, renal cell carcinoma, apoptosis, upregulated miRNA advanced-stage RCC patients is extremely poor (5-10\%) due to recurrence or distant metastasis (2). At diagnosis, nearly $30 \%$ of RCC patients present with metastasis (3). Current treatments for RCC include molecular-targeted therapeutics such as anti-angiogenic multi-tyrosine kinase inhibitors or mTOR inhibitors, which are being widely used for patients with metastatic or recurrent RCC. However, these types of therapies are not expected to have curative effects as they only slightly extend progression-free survival (4). Therefore, to improve the outcome of patients with RCC, it is necessary to fully elucidate the molecular mechanisms underlying the development and progression of RCC and the associated oncogenic pathways.

The discovery of non-coding RNAs (ncRNAs) in the human genome was an important conceptual breakthrough, and the understanding of ncRNAs is important for progress in cancer research. MicroRNAs (miRNAs) are endogenous small ncRNA molecules (19-22 bases in length) that regulate proteincoding gene expression by repressing translation or cleaving RNA transcripts in a sequence-specific manner (5). miRNAs are predicted to regulate more than $60 \%$ of the protein-coding genes in the human genome (6). Accumulating evidence suggests that miRNAs are aberrantly expressed in many human cancers and play significant roles in human oncogenesis and metastasis (7). Therefore, identifying aberrantly expressed miRNAs is an important first step to elucidate the details of miRNA-mediated oncogenic pathways in cancer cells.

Previously, our miRNA expression signature of ccRCC revealed that microRNA-1274a (miR-1274a) was significantly upregulated in cancer tissues, suggesting that this miRNA functions as an oncogenic miRNA (8). Therefore, the aim of the present study was to investigate the functional significance of $m i R-1274 a$ and to identify the molecular targets and pathways mediated by $m i R-1274 a$ in ccRCC cells. Our data demonstrated that downregulation of $m i R-1274 a$ inhibited cancer cell proliferation and induced apoptosis. Moreover, gene expression data and in silico database analysis showed that bone morphogenetic protein receptor type $1 \mathrm{~B}$ (BMPR1B) gene was identified as a candidate target of $m i R-1274 a$. The discovery of genes and pathways mediated by oncogenic $m i R-1274 a$ provides important insights into the potential mechanisms of ccRCC oncogenesis and suggests novel therapeutic strategies for the treatment of ccRCC. 


\section{Materials and methods}

Clinical specimens and cell culture. The ccRCC and adjacent normal tissues were collected from 40 ccRCC patients immediately after undergoing radical nephrectomies at Kagoshima University Hospital between 2003 and 2013. The clinicopathological information of the patients is shown in Table I. The specimens were staged according to the American Joint Committee on Cancer-Union Internationale Contre le Cancer (UICC) tumor-node-metastasis (TNM) classification and histologically graded (9). This study was approved by the Bioethics Committee of Kagoshima University; prior written informed consent and approval were obtained from all patients. We used human ccRCC cell lines, 7860, A498, ACHN and Cakil, obtained from the American Type Culture Collection (ATCC; Manassas, VA, USA). These cell lines were incubated in RPMI-1640 medium (Thermo Fisher Scientific, Waltham, MA, USA) supplemented with $10 \%$ fetal bovine serum (FBS) and maintained in humidified incubators $\left(5 \% \mathrm{CO}_{2}\right)$ at $37^{\circ} \mathrm{C}$.

Tissue collection and RNA extraction. Tissues were immersed in RNAlater (Thermo Fisher Scientific) and stored at $-20^{\circ} \mathrm{C}$ until RNA extraction was conducted. Total RNA, including miRNA, was extracted using the mirVana ${ }^{\mathrm{TM}}$ miRNA isolation kit (Thermo Fisher Scientific) following the manufacturer's protocol.

Quantitative real-time reverse transcriptase-polymerase chain reaction ( $q R T-P C R$ ). Stem-loop RT-PCR (TaqMan MicroRNA Assays; product ID: 2883 for miR-1274a; Applied Biosystems, Foster City, CA, USA) was used to quantify miRNAs according to the manufacturer's protocol for PCR conditions. We used human RNU48 (product ID: 001006; Applied Biosystems) as internal control. For investigating the genes, we applied SYBR-Green quantitative PCR-based array approach, and the following primers were used: $B M R P 1 B$ forward primer, 5'-CTTTTGCGAAGTGCAGGAAAAT-3' and reverse primer, 5'-TGTTGACTGAGTCTTCTGGAC AA-3'; GUSB forward primer, 5'-CGTCCCACCTAGAAT CT GCT-3' and reverse primer, 5'-TTGCTCACAAAGGTCAC AGG-3'. qRT-PCR was performed with $500 \mathrm{ng}$ of total RNA using the Power SYBR-Green Master Mix (cat. no. 4367659; Applied Biosystems) on the 7300 Real-Time PCR System (Applied Biosystems). The experimental procedures were conducted according to the protocol recommended by the manufacturer. The specificity of amplification was monitored using the dissociation curve of the amplified product. $B M P R I B$ data values were normalized to GUSB. The $\Delta \Delta \mathrm{Ct}$ method was employed to calculate the fold-change of expression level relative to the internal controls.

miRNA inhibitor transfection. ccRCC cell lines were transfected with Lipofectamine RNAiMAX transfection reagent and Opti-MEM (Thermo Fisher Scientific) with 10-60 nM miRNA inhibitor (hsa-miR-1274a; product ID: AM13432, cat. no. AM17000) and negative control \#1 anti-miRNA (cat. no. AM17010; Thermo Fisher Scientific).

Cell proliferation and apoptosis assays following antimiR-1274a transfection. Seventy-two hours after transfection, cell proliferation was determined with an XTT assay using
Table I. Clinicopathological characteristics of the ccRCC patients.

\begin{tabular}{lc}
\hline Characteristics & Data \\
\hline Total number of cases & 40 \\
Mean age \pm SD (years) & $64.4 \pm 13.5$ \\
Sex, $\mathrm{n}(\%)$ & \\
Male & $14(35.0)$ \\
Female & $26(65.0)$ \\
Stage, $\mathrm{n}(\%)$ & \\
pT1a & $18(45.0)$ \\
pT1b & $13(32.5)$ \\
pT2 & $2(5.0)$ \\
pT3a & $4(10.0)$ \\
pT3b & $3(7.5)$ \\
pT4 & $0(0.0)$ \\
Grade, $\mathrm{n}(\%)$ & \\
G1 & $8(20.0)$ \\
G2 & $30(75.0)$ \\
G3 & $0(0.0)$ \\
Unknown & $2(5.0)$ \\
Infiltration, $n(\%)$ & \\
$\alpha$ & $27(67.5)$ \\
$\beta$ & $13(32.5)$ \\
$\gamma$ & $0(0.0)$ \\
Venous invasion, $\mathrm{n}(\%)$ & \\
v (-) & $29(72.5)$ \\
v (+) & $11(27.5)$ \\
\hline
\end{tabular}

the Cell Proliferation Kit II (Roche Molecular Biochemicals, Mannheim, Germany) according to the manufacturer's instructions. Cell apoptosis assays were carried out using ccRCC cell lines transfected with the transfection reagent, anti-miR-control and anti-miR-1274a, in 6-well tissue culture plates. Cells were harvested $72 \mathrm{~h}$ after transfection by trypsinization and washed in cold phosphate-buffered saline (PBS). Double staining with FITC-Annexin V and propidium iodine (PI) was carried out using the FITC Annexin V apoptosis detection kit (BD Biosciences, Bedford, MA, USA) according to the manufacturer's recommendations and analyzed within $1 \mathrm{~h}$ by flow cytometry (FACScan; BD Biosciences). Cells were discriminated into viable cells, dead cells, early and late apoptotic cells by CellQuest software (BD Biosciences), and then the percentages of total apoptotic cells from each samples were compared. Experiments were conducted in triplicate.

Putative target gene analysis of miR-1274a. To investigate the expression status of candidate miR-1274a target genes in ccRCC clinical specimens, we examined Oligo Microarray human 44K (Agilent Technologies, Santa Clara, CA, USA) expression profiling of ACHN with anti-miR-control and anti-miR-1274a transfectants. Then, gene expression data were adapted to Kyoto Encyclopaedia of Genes and Genomes (KEGG) pathway categories by the GENECODIS program 

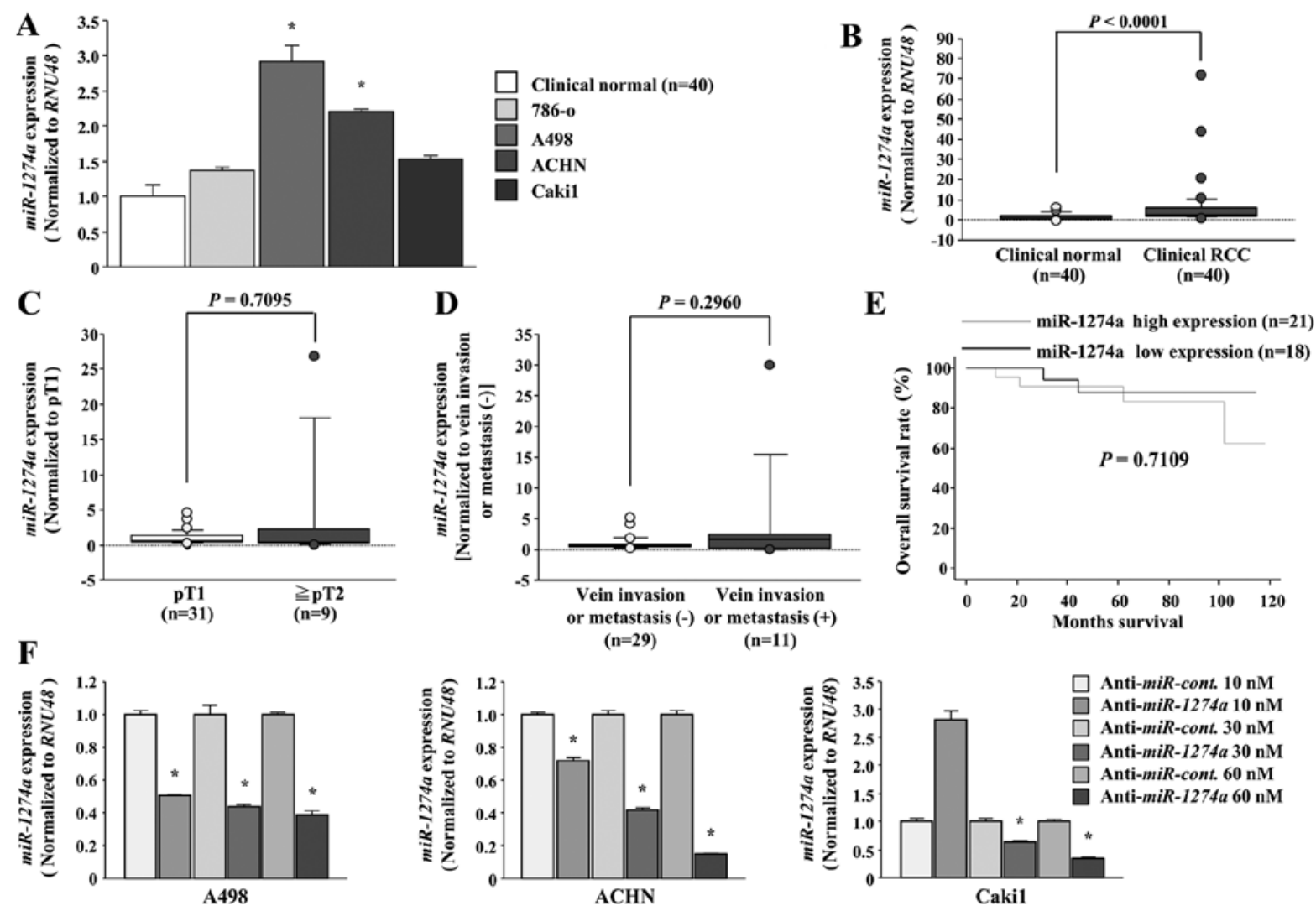

Figure 1. Expression levels of $m i R-1274 a$ and knockdown efficiency of anti-miR-1274a transfection in ccRCC cells. (A) Expression levels of $m i R-1274 a$ in normal kidney tissues and ccRCC cell lines, and (B) in normal kidney tissues and ccRCC specimens, were determined by qRT-PCR. Data were normalized to RNU48 expression ("P<0.05). (C and D) Expression levels of $m i R-1274 a$ in ccRCC patients (C) with $\mathrm{pT} 1$ and $\geq \mathrm{pT} 2$, and (D) without vein invasion or metastasis and with vein invasion or metastasis. (E) Kaplan-Meier survival curves for overall survival according to miR-1274a expression in $39 \mathrm{ccRCC}$ patients. P-values were calculated using the log-rank test. (F) The expression level of miR-1274a in A498, ACHN and Cakil cells $48 \mathrm{~h}$ after transfection with 10-60 nM antimiR-1274a $($ 'P<0.05).

(http://genecodis.cnb.csic.es/). Publicly available expression data from ccRCC specimens in the GEO database (accession number: GSE36895 + GSE22541) were used. We merged these data sets and selected putative genes targeted by $m i R-1274 a$ according to the TargetScan algorithm (June, 2011 release, http://www.targetscan.org/vert_50/).

Plasmid construction and Dual-Luciferase reporter assays. A partial wild-type sequence of the BMPR1B 3'-untranslated region (UTR) or the same region lacking its $m i R-1274 a$ target site (positions 3237-3243 of the BMPR1B 3'-UTR, according to the TargetScan program) was inserted between the $X h o I$ and PmeI restriction sites in the 3'-UTR of the hRluc gene in the psi-CHECK-2 vector (C8021; Promega, Madison, WI, USA). ACHN cells were transfected with $50 \mathrm{ng}$ of the vector and $10 \mathrm{nM} m i R-1274 a$ (product ID: AM13432, cat. no. AM17100; Thermo Fisher Scientific) or control miRNAs (product ID: AM17111; Thermo Fisher Scientific) using Lipofectamine 2000 (Invitrogen). The activities of firefly and Renilla luciferases in cell lysates were determined with a Dual-Luciferase assay system (E1960; Promega). Normalized data were calculated as the ratio of Renilla/firefly luciferase activities.

Overall survival analysis of a ccRCC cohort (KIRC) from The Cancer Genome Atlas (TCGA). The TCGA cohort database for 72 normal kidneys and $534 \mathrm{ccRCC}$ patients (KIRC) was used to determine the relationship between $B M P R 1 B$ expression in normal and in ccRCCs, and between BMPRIB expression of ccRCC patients and overall survival. Normalized RNA-seq by expectation-maximization (RSEM) value from RNA-seq expression data were used for gene expression quantification (10). Full sequencing information and clinical information were acquired from the cBioPortal (http://www.cbioportal. org/public-portal/) and the TCGA (https://tcga-data.nci.nih. gov/tcga/) (11-13).

Immunohistochemistry. A tissue microarray of $67 \mathrm{ccRCC}$ samples and 10 normal renal tissues was obtained from US Biomax, Inc. (KD806; Rockville, MD, USA). Immunostaining was carried out on the tissue microarray according to the manufacturer's protocol using the UltraVision Detection System (Thermo Fisher Scientific). Primary rabbit polyclonal antibodies against BMPR1B (GTX102453; GeneTex, Inc., Irvine, CA, USA) were diluted 1:500. Slides were treated with biotinylated goat anti-rabbit. Diaminobenzidine hydrogen peroxidase was the chromogen and counterstaining was conducted with $0.5 \%$ hematoxylin. Immunostaining was evaluated according to a previously described scoring method (14).

Statistical analysis. Relationships between two or three variables and numerical values were analyzed using the Mann-Whitney U test or Bonferroni-adjusted Mann-Whitney 
A

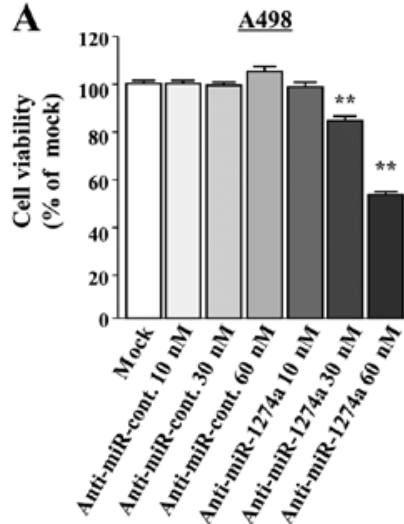

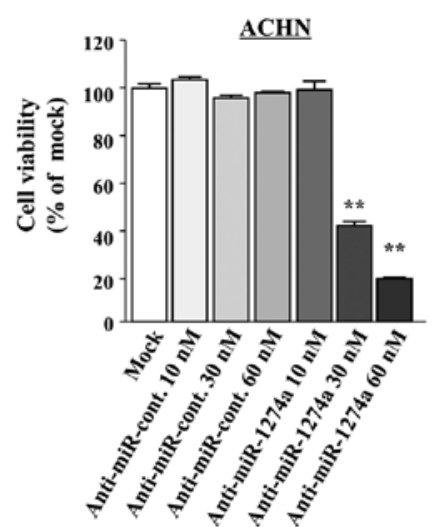

Caki1

B
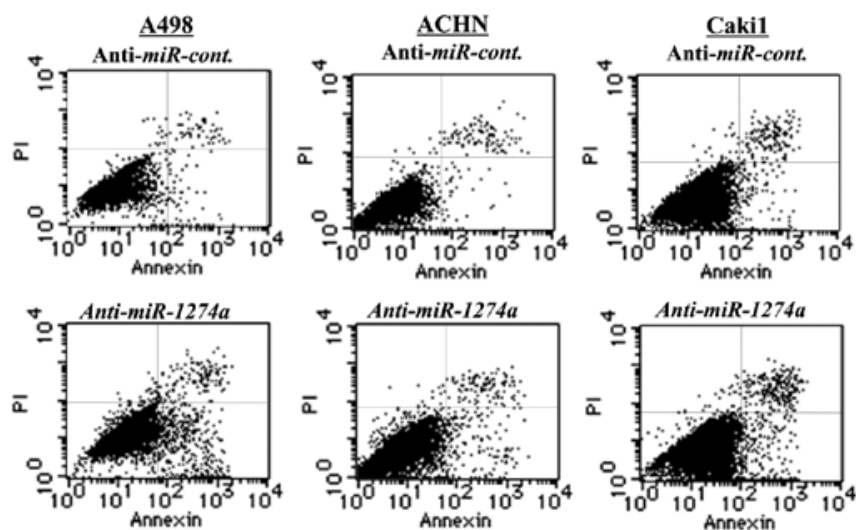

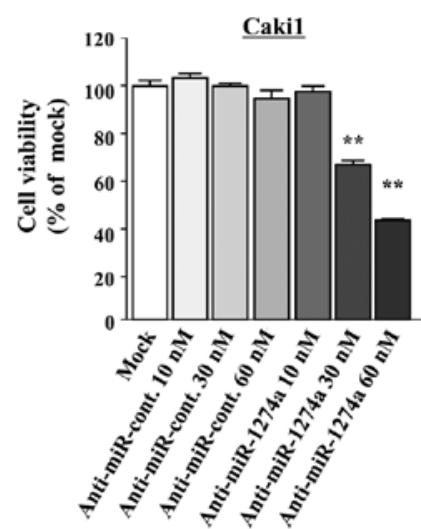

Figure 2. Effects of anti-miR-1274a on cell growth and apoptosis. (A) Cell growth was determined by XTT assays in the A498, ACHN and Cakil cells 72 h after

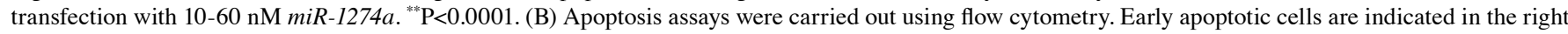
bottom quadrant and late apoptotic cells are indicated in the right upper quadrant. The normalized ratios of apoptotic cells are shown in the histograms $($ P $<0.05)$.

$\mathrm{U}$ test. Overall survival of ccRCC patients from the TCGA cohort was evaluated by the Kaplan-Meier method. Patients were numerically divided equally into two groups according to $B M P R 1 B$ expression, and the differences between the two groups were evaluated by log-rank tests. We used Expert StatView software, version 5.0 (SAS Institute, Inc., Cary, NC, USA) for these analyses.

\section{Results}

Expression levels of miR-1274a in ccRCC specimens and ccRCC cell lines. We evaluated the expression level of $m i R-1274 a$ in ccRCC tissues $(\mathrm{n}=40)$, adjacent normal kidney tissue $(\mathrm{n}=40)$ and ccRCC cell lines (786-O, A498, ACHN and Caki1). The expression levels of $m i R-1274 a$ were significantly higher in tumor tissues and two ccRCC cell lines (A498 and $\mathrm{ACHN})$ than in normal kidney tissue $(\mathrm{P}<0.05$, Fig. $1 \mathrm{~A}$, and $\mathrm{P}<0.0001$, Fig. 1B). miR-1274a expression in Caki1 was higher than that noted in the normal kidneys even though there was no significant difference. Therefore, we used A498, ACHN and Cakil cells for the next experiments. There were no significant correlations between any of the clinicopathological parameters or overall survival rate and the expression of $m i R-1274 a$ (Fig. 1C-E). Next, we evaluated the miR-1274a expression level following anti-miR-1274a transfection. The expression of miR-1274a in the A498, ACHN and Cakil cells was sufficiently downregulated following transfection of 30 and $60 \mathrm{nM}$ anti-miR-1274a (P<0.05; Fig. 1F).
Effects of the inhibition of miR-1274a on cell proliferation and apoptosis in ccRCC cell lines. To examine the functional roles of $m i R-1274 a$, we performed loss-of-function studies by using anti-miRNA-transfected A498, ACHN and Cakil cells. XTT assays revealed that there was a significant inhibition of cell proliferation in the 10, 30 and $60 \mathrm{nM}$ anti-miR-1274a-transfectants $(\mathrm{P}<0.0001$; Fig. 2A). We also analyzed the numbers of apoptotic cells and found that miR-1274a inhibition significantly induced apoptosis of the A498, ACHN and Cakil cells $(\mathrm{P}<0.05$; Fig. 2B).

Screening of putative target genes of miR-1274a in ccRCC. To gain further insight into the molecular mechanisms regulated by oncogenic miR-1274a in ccRCC cells, we screened $m i R$-1274a-regulated genes by using in silico and genome-wide gene expression analysis (Fig. 3). As for the target search by oligo microarray, we used ACHN as the knockdown efficiency and cell proliferative inhibition rate by anti-miRNA was the strongest in the ACHN cells. First, we found that 694 genes were upregulated in the oligo microarray by anti-miR-1274a-transfectants of ACHN. These 694 genes were assigned KEGG annotations using singular enrichment analysis of GeneCodis to identify the molecular pathways regulated by $m i R-1274 a$. Twenty signaling pathways and 73 genes were identified in this analysis (Table II). Furthermore, we examined gene expression signatures that were downregulated in clinical ccRCC specimens from the GEO database and found that 9 genes were significantly downregulated in 
Table II. Significantly enriched annotations regulated by miR-1274a (top 20 pathways).

\begin{tabular}{|c|c|c|c|}
\hline $\begin{array}{l}\text { No. of } \\
\text { genes }\end{array}$ & P-value & KEGG pathway & Genes \\
\hline 12 & $1.35 \mathrm{E}-08$ & NOD-like receptor signaling pathway & $\begin{array}{l}\text { IL6, BIRC2, CXCL2, TNFAIP3, NFKBIA, CXCL1, BIRC3, } \\
\text { IL8, MAPK9, CARD9, CCL2, NLRP3 }\end{array}$ \\
\hline 19 & $3.52 \mathrm{E}-06$ & Cytokine-cytokine receptor interaction & $\begin{array}{l}\text { IL12RB1, IL6, TNFRSF10D, CXCL2, BMPR1B, CXCL3, } \\
\text { FAS, CXCL1, CCL20, CNTF, CXCL16, IL8, EDA2R, CCL2, } \\
\text { LTB, IL18RAP, TNFRSF9, VEGFA, CSF2 }\end{array}$ \\
\hline 11 & $5.04 \mathrm{E}-06$ & Rheumatoid arthritis & $\begin{array}{l}\text { IL6, FOS, CXCL1, CCL20, IL8, ICAM1, CCL2, LTB, VEGFA, } \\
\text { CSF2, MMP1 }\end{array}$ \\
\hline 19 & 5.47E-05 & Pathways in cancer & $\begin{array}{l}\text { IL6, MDM2, FOS, BIRC2, NFKBIA, ITGA2, FAS, ETS1, } \\
\text { BIRC3, RUNX1, IL8, MAPK9, LAMB3, MECOM, TRAF1, } \\
\text { VEGFA, WNT1, STAT5A, MMP1 }\end{array}$ \\
\hline 16 & $1.74 \mathrm{E}-04$ & $\begin{array}{l}\text { MAPK signaling pathway } \\
\text { (American trypanosomiasis) }\end{array}$ & $\begin{array}{l}\text { GADD45A, NLK, FOS, DUSP1, DUSP16, RELB, DDIT3, } \\
\text { FAS, PPM1A, HSPA6, CACNA1B, DUSP5, MAPK9, } \\
\text { MECOM, DUSP10, MAP3K8 }\end{array}$ \\
\hline 9 & $8.25 \mathrm{E}-04$ & $\begin{array}{l}\text { Chagas disease } \\
\text { (American trypanosomiasis) }\end{array}$ & $\begin{array}{l}\text { IL6, FOS, NFKBIA, FAS, SERPINE1, IL8, MAPK9, CCL2, } \\
\text { GNA15 }\end{array}$ \\
\hline 10 & $9.08 \mathrm{E}-04$ & Osteoclast differentiation & $\begin{array}{l}\text { FOS, SIRPG, NFKBIA, RELB, SQSTM1, LILRB3, SOCS3, } \\
\text { FOSB, MAPK9, JUNB }\end{array}$ \\
\hline 8 & $6.08 \mathrm{E}-03$ & $\mathrm{~T}$ cell receptor signaling pathway & $\begin{array}{l}\text { FOS, NFKBIA, PAK6, MAPK9, NFKBIE, PTPRC, CSF2, } \\
\text { MAP3K8 }\end{array}$ \\
\hline 7 & $8.12 \mathrm{E}-03$ & ErbB signaling pathway & NRG1, EREG, PAK6, ABL2, HBEGF, MAPK9, STAT5A \\
\hline 10 & $1.29 \mathrm{E}-02$ & Chemokine signaling pathway & $\begin{array}{l}\text { CXCL2, NFKBIA, CXCL3, CXCL1, CCL20, CXCL16, IL8, } \\
\text { GRK5, CCL2, GNGT1 }\end{array}$ \\
\hline 7 & $1.62 \mathrm{E}-02$ & Toll-like receptor signaling pathway & IL6, FOS, NFKBIA, IL8, IRF5, MAPK9, MAP3K8 \\
\hline 6 & $2.49 \mathrm{E}-02$ & TGF- $\beta$ signaling pathway & CHRD, BMPR1B, BMP6, ID3, SMURF1, ACVR1C \\
\hline 6 & $2.60 \mathrm{E}-02$ & Small cell lung cancer & BIRC2, NFKBIA, ITGA2, BIRC3, LAMB3, TRAF1 \\
\hline 6 & $2.72 \mathrm{E}-02$ & Apoptosis & TNFRSF10D, BIRC2, NFKBIA, FAS, BIRC3, IRAK2 \\
\hline 4 & $3.84 \mathrm{E}-02$ & Bladder cancer & MDM2, IL8, VEGFA, MMP1 \\
\hline 5 & 4.01E-02 & p53 signaling pathway & MDM2, GADD45A, FAS, SERPINE1, ZMAT3 \\
\hline 5 & 4.01E-02 & $\begin{array}{l}\text { Epithelial cell signaling in } \\
\text { Helicobacter pylori infection }\end{array}$ & NFKBIA, CXCL1, HBEGF, IL8, MAPK9 \\
\hline 7 & 4.09E-02 & Measles & IL6, TNFRSF10D, TNFAIP3, NFKBIA, FAS, HSPA6, STAT5A \\
\hline 5 & $4.62 \mathrm{E}-02$ & Chronic myeloid leukemia & MDM2, NFKBIA, RUNX1, MECOM, STAT5A \\
\hline 6 & 4.63E-02 & Amoebiasis & IL6, CXCL1, IL8, LAMB3, GNA15, CSF2 \\
\hline
\end{tabular}

53 ccRCCs compared to 23 normal kidney tissues. Among these, we focused on BMPR1B, as the BMPR1B expression level in ccRCC tissues was most significantly downregulated compared with the level noted in normal tissues (Table III) and TargetScan 5.2 database predicted that there was a binding site for $m i R-1274 a$ in BMPRIB 3'UTR.

$B M P R 1 B$ is regulated by $m i R-1274 a$. We performed qRT-PCR to determine whether inhibition of $m i R-1274 a$ resulted in upregulation of $B M P R 1 B$ mRNA expression in ccRCC cells. The mRNA levels of $B M P R 1 B$ were significantly upregulated in the anti-miR-1274a transfectants in comparison with the anti-miR-control transfectants in ACHN and Caki1 cells
$(\mathrm{P}<0.05$; Fig. 4A). Next, we performed luciferase reporter assays to determine whether the 3'-UTR of BMPR1B contained an actual binding site of $m i R-1274 a$. The TargetScan database predicted that one putative $m i R-1274 a$ binding site existed in the 3'-UTR of BMPRIB (positions 3237-3243; Fig. 4B). We found that the luminescence intensity in ACHN cells was significantly reduced by transfection of $m i R-1274 a$ with the wild-type vector carrying the 3'-UTR of $B M P R 1 B$, whereas transfection with a deletion vector showed no decrease in luminescence $(\mathrm{P}<0.05$; Fig. 4B). The expression levels of BMPR1B were significantly lower in ccRCCs than in normal kidneys using our clinical samples $(\mathrm{P}<0.0001$; Fig. 4C). These data were consistent with the in silico database analysis 

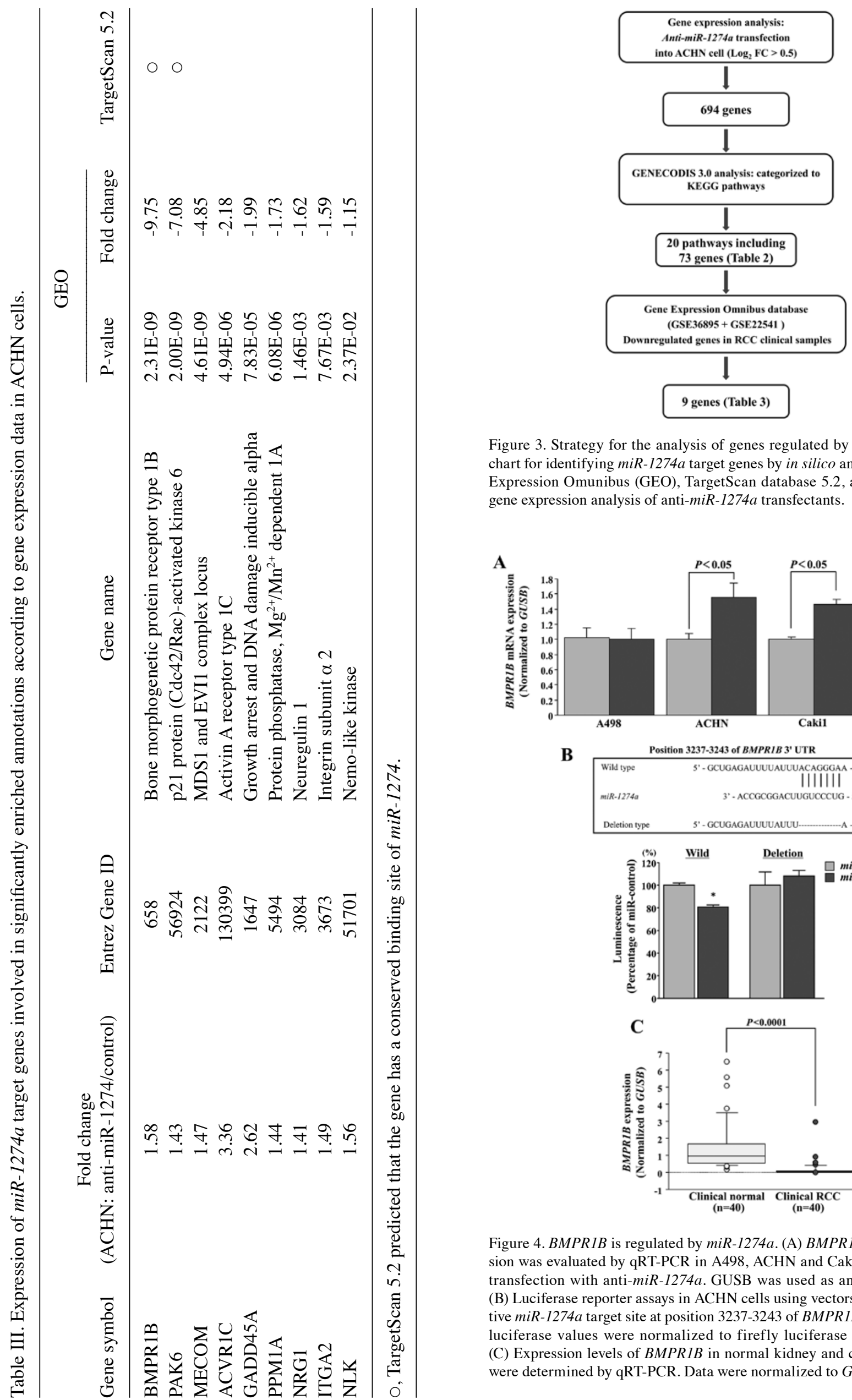

Figure 3. Strategy for the analysis of genes regulated by miR-1274a. Flow chart for identifying $m i R-1274 a$ target genes by in silico analysis using Gene Expression Omunibus (GEO), TargetScan database 5.2, and genome-wide gene expression analysis of anti-miR-1274a transfectants.

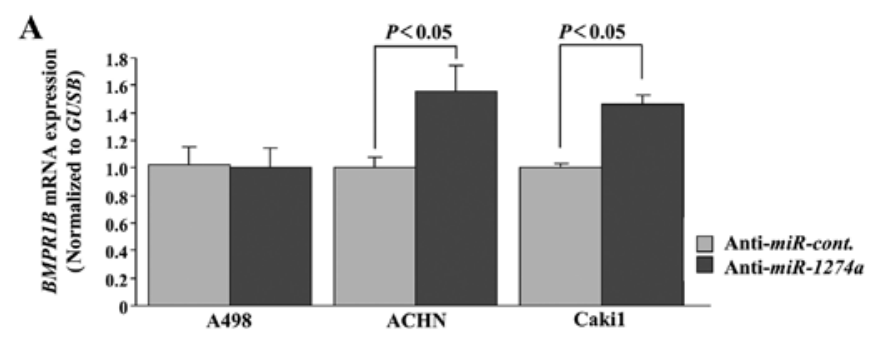

B
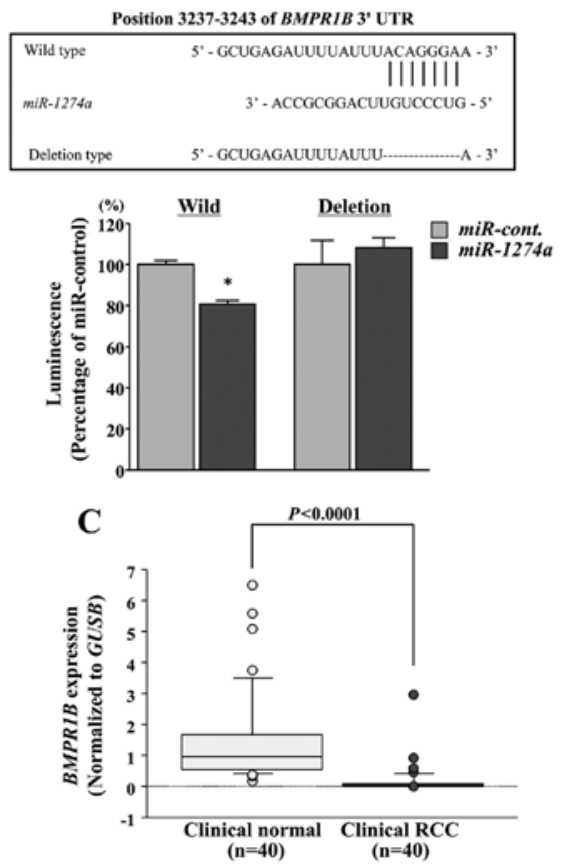

Figure 4. BMPR1B is regulated by $m i R-1274 a$. (A) BMPR1B mRNA expression was evaluated by qRT-PCR in A498, ACHN and Cakil cells $72 \mathrm{~h}$ after transfection with anti-miR-1274a. GUSB was used as an internal control. (B) Luciferase reporter assays in ACHN cells using vectors encoding a putative miR-1274a target site at position 3237-3243 of BMPR1B 3'-UTR. Renilla luciferase values were normalized to firefly luciferase values $(" \mathrm{P}<0.05)$. (C) Expression levels of BMPR1B in normal kidney and ccRCC specimens were determined by qRT-PCR. Data were normalized to GUSB expression. 

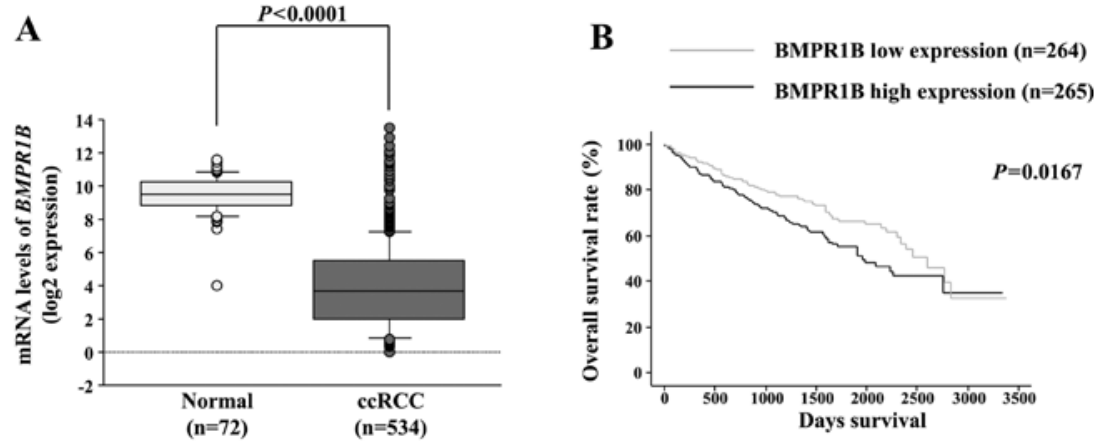

Figure 5. Analysis of TCGA kidney clear cell carcinoma (KIRC) datasets. (A) Expression levels of BMPRIB in normal kidney tissues and ccRCC tissue specimens were determined by the KIRC dataset. (B) Kaplan-Meier survival curves for overall survival rate according to BMPRIB expression in $529 \mathrm{ccRCC}$ patients. P-values were calculated using the log-rank test.
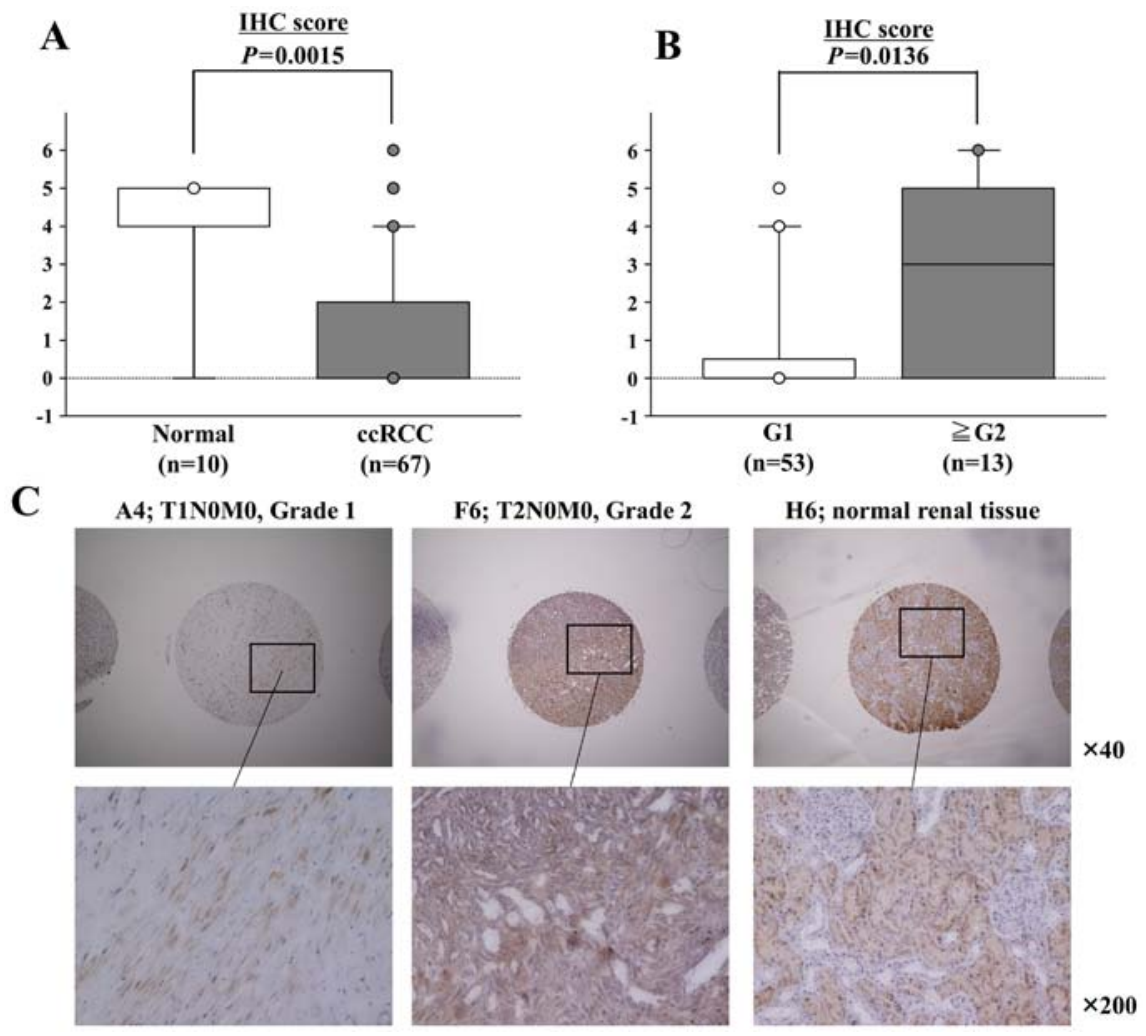

Figure 6. Immunohistochemical staining of BMPR1B in tissue specimens. (A) Expression levels of BMPR1B were significantly lower in 67 ccRCCs in comparison with 10 normal renal tissues, (B) and in ccRCCs with G1 $(n=53)$ in comparison with ccRCCs with G2 or higher $(n=13)$. (C) Representative images of immunohistochemical staining of BMPR1B in tissue specimens (magnification, $\mathrm{x} 40$ and $\mathrm{x} 200$ ).

(Fig. 3) indicating that downregulated BMPR1B as reported in the GEO data analysis was upregulated when the cells were treated with anti-miR-1274a in the microarray.

BMPRIB expression as a prognostic marker of $c c R C C$. The expression levels of $B M P R I B$ were significantly lower in ccRCC tissue samples than that noted in the normal kidneys using the TCGA database $(\mathrm{P}<0.0001$; Fig. $5 \mathrm{~A})$. We then evaluated the correlation of $B M P R I B$ expression levels with overall survival of the ccRCC patients. The cohort was numerically divided equally into two groups based on $B M P R I B$ expression. Unexpectedly, we found that the low $B M P R 1 B$ expression group $(n=264)$ had better median overall survival in comparison with the high BMPR1B expression group $(n=265)(P=0.0167$;
Fig. 5B). This findings were supported by the results of the immunohistochemical staining of BMPR1B in tissue specimens (Fig. 6). The expression scores of BMPR1B were significantly lower in $67 \mathrm{ccRCCs}$ in comparison with 10 normal renal tissues $(\mathrm{P}=0.0015$; Fig. 6A). On the other hand, the scores were significantly higher in ccRCCs with high-grade tumors (more than $\mathrm{G} 2, \mathrm{n}=13$ ) in comparison with scores in ccRCCs with low-grade tumors $(\mathrm{G} 1, \mathrm{n}=53)(\mathrm{P}=0.0136$; Fig. 6B).

\section{Discussion}

There is considerable evidence that normal miRNA regulatory mechanisms are disrupted in cancer cells (7). However, the regulatory mechanisms by which upregulated miRNAs 
exert their effects has not been fully understood compared to downregulated miRNAs. Upregulated miRNAs have been mainly viewed as markers (15), and functional studies have not been performed in detail, even though many studies have successfully inhibited miRNA expression using miRNA inhibitors, morpholinos, sponges, or CRISPR/Cas9 $(8,16-18)$. One of the possible reasons is that miRNA gain-of-function studies in downregulated miRNAs can be easily performed by transfection of precursor miRNAs with sufficient efficiency. In the present study, we suppressed upregulated $m i R-1274 a$ with miRNA antisense inhibitor. Even though we obtained nearly $85 \%$ knockdown efficiency at most, high concentration of the miRNA antisense inhibitor was needed. Therefore, other approaches such as CRISPR/Cas9 to gain adequate knockdown efficiency without off-target effects will be promising in subsequent research.

Our previous studies of miRNA expression signatures in ccRCC identified several upregulated miRNAs ( $m i R-885-5 p$, $m i R-1274 a, m i R-210-3 p, m i R-224$ and $m i R-1290$ ) (8). In this study, we focused on $m i R-1274 a$, as increased expression of $m i R-1274 a$ has been reported in other types of human cancers $(19,20)$. Wang et al (19) reported that $m i R-1274 a$ was overexpressed in gastric cancer tissues or cancer cell lines, and that $m i R-1274 a$ promoted proliferation, migration and epithelial-mesenchymal transition (EMT) by suppressing FOXO4, which is a transcriptional factor and leads to cell cycle arrest or apoptosis. In melanoma, high expression of $m i R-1274 a$ was also reported (20). On the other hand, contrasting roles of $m i R-1274 a$ have also been reported in cancers to date. Zhou et al (21) reported that $m i R-1274 a$ was upregulated by sorafenib, which is a multi-kinase inhibitor clinically used for cancer treatment. They also found that $m i R-1274 a$ could significantly suppress one of the protease proteins ADAM9 and accelerate antitumor immunity in hepatocellular carcinoma (HCC). Yan et al (22) also reported similar results in HCC where miR-1274a was upregulated by paclitaxel. In fields other than cancers, Ezzie et al (23) reported that $m i R-1274 a$ was one of the highly expressed miRNAs in lungs from subjects with chronic obstructive pulmonary disease (COPD) compared with smokers without COPD. Thus, diverse types of roles for $m i R-1274 a$ have been reported not only in cancers but also in chronic disease.

In the present study, we investigated the pathways and targets that were regulated by oncogenic $m i R-1274 a$ in ccRCC cells. In the process of the identification of $m i R-1274 a$-regulated molecular pathways and target genes, we used a combination of expression data and in silico database analysis (24). Using this strategy, we have identified molecular targets and pathways in several types of cancer that are regulated by tumor-suppressive miRNAs. In this study, 'Cytokine-cytokine receptor interaction' and 'TGF- $\beta$ signaling pathway' were significantly selected as candidate pathways regulated by $m i R-1274 a$ in ccRCC cells. In these pathways, BMPR1B was selected as a candidate target of $m i R-1274 a$. BMPR1B is a member of the BMP receptor family of transmembrane serine/threonine kinases, and the ligands of this receptor are BMPs $(25,26)$. BMPs, members of the TGF- $\beta$ superfamily, bind their receptor including BMPR1B, which lead to activation of the SMADdependent pathway subsequently through phosphorylation of SMAD1/5/8 $(25,26)$. Then phosphorylated SMADs form a complex with the common signaling transducer SMAD4, resulting in the induction of apoptosis (26-30). Therefore, our finding that suppression of $m i R-1274 a$ caused apoptosis through BMPR1B upregulation is plausible. However, A498 ccRCC cells did not exhibit increased $B M P R 1 B$ expression, even though ACHN and Cakil cells had rescued expression, and $m i R-1274 a$ suppression in all 3 cell lines induced apoptosis in this study. Even though low expression of BMPR1B was observed in ccRCC compared to normal kidneys, BMPR1B expression was significantly higher in high-grade ccRCCs in comparison with low-grade ccRCCs as detected by immunohistochemistry. In addition, the low expression patient group had prolonged median overall survival in comparison with the high expression patient group according to the TCGA analyses. BMPR1B upregulation might be an early event in ccRCC carcinogenesis, and its functional changes might have occurred as the tumor acquires malignant potential at a late stage. Further study is necessary to elucidate the further functional role of BMPR1B. Concerning $m i R-1274 a$, we did not find any significant correlation between expression of $m i R$ $1274 a$ and any of the clinicopathological parameters including prognosis. This may be due to the small sample numbers and short follow-up periods. A larger scale and longer follow-up period of study should be considered in future research.

In conclusion, upregulation of $m i R-1274 a$ was observed in ccRCC clinical specimens and cell lines. Suppression of miR-1274a significantly inhibited cancer cell proliferation through apoptosis, suggesting that $m i R-1274 a$ functions as an oncogenic miRNA in ccRCC cells. The identification of tumor-suppressive $B M P R 1 B$ regulated by $m i R-1274 a$ may lead to a better understanding of ccRCC oncogenesis and the development of new therapeutic strategies to treat this disease.

\section{Acknowledgements}

We thank Mutsumi Miyazaki for the excellent laboratory assistance and the critical editing of this manuscript. The present study was supported by the KAKENHI (B) 16H05464, the KAKENHI (C) 16K11015 and 17K11148, the KAKENHI (HOUGA) 16K15691, and the KAKENHI (WAKATE)17K16799.

\section{References}

1. Ljungberg B, Bensalah K, Canfield S, Dabestani S, Hofmann F, Hora M, Kuczyk MA, Lam T, Marconi L, Merseburger AS, et al: EAU guidelines on renal cell carcinoma: 2014 update. Eur Urol 67: 913-924, 2015.

2. Hadoux J, Vignot S and De La Motte Rouge T: Renal cell carcinoma: Focus on safety and efficacy of temsirolimus. Clin Med Insights Oncol 4: 143-154, 2010.

3. Gupta K, Miller JD, Li JZ, Russell MW and Charbonneau C: Epidemiologic and socioeconomic burden of metastatic renal cell carcinoma (mRCC): A literature review. Cancer Treat Rev 34: 193-205, 2008.

4. Margulis V, Master VA, Cost NG, Leibovich BC, Joniau S, Kuczyk M, Mulders PF, Kirkali Z, Wirth MP, Hirao Y, et al: International consultation on urologic diseases and the European Association of Urology international consultation on locally advanced renal cell carcinoma. Eur Urol 60: 673-683, 2011.

5. Bartel DP: MicroRNAs: Genomics, biogenesis, mechanism, and function. Cell 116: 281-297, 2004.

6. Friedman RC, Farh KK, Burge CB and Bartel DP: Most mammalian mRNAs are conserved targets of microRNAs. Genome Res 19: 92-105, 2009. 
7. Calin GA and Croce CM: MicroRNA signatures in human cancers. Nat Rev Cancer 6: 857-866, 2006.

8. Yoshino H, Yonemori M, Miyamoto K, Tatarano S, Kofuji S, Nohata N, Nakagawa $M$ and Enokida H: microRNA-210-3p depletion by CRISPR/Cas9 promoted tumorigenesis through revival of TWIST1 in renal cell carcinoma. Oncotarget 8 : 20881-20894, 2017.

9. Sobin LH, Gospodarowicz MK and Wittenkind C: TNM Classification of Malignant Tumour. 7th edition. Wiley-Liss Inc., New York, pp255-257, 2009.

10. Li B and Dewey CN: RSEM: Accurate transcript quantification from RNA-Seq data with or without a reference genome. BMC Bioinformatics 12: 323, 2011.

11. Creighton CJ, Morgan M, Gunaratne PH, Wheeler DA, Gibbs RA, Gordon Robertson A, Chu A, Beroukhim R, Cibulskis K, Signoretti S, et al; Cancer Genome Atlas Research Network: Comprehensive molecular characterization of clear cell renal cell carcinoma. Nature 499: 43-49, 2013.

12. Cerami E, Gao J, Dogrusoz U, Gross BE, Sumer SO, Aksoy BA, Jacobsen A, Byrne CJ, Heuer ML, Larsson E, et al: The cBio cancer genomics portal: An open platform for exploring multidimensional cancer genomics data. Cancer Discov 2: 401-404, 2012.

13. Gao J, Aksoy BA, Dogrusoz U, Dresdner G, Gross B, Sumer SO Sun Y, Jacobsen A, Sinha R, Larsson E, et al: Integrative analysis of complex cancer genomics and clinical profiles using the cBioPortal. Sci Signal 6: pl1, 2013.

14. Yoshino H, Chiyomaru T, Enokida H, Kawakami K, Tatarano S, Nishiyama K, Nohata N, Seki N and Nakagawa M: The tumoursuppressive function of miR-1 and miR-133a targeting TAGLN2 in bladder cancer. Br J Cancer 104: 808-818, 2011.

15. Iorio MV and Croce CM: MicroRNA dysregulation in cancer: Diagnostics, monitoring and therapeutics. A comprehensive review. EMBO Mol Med 4: 143-159, 2012.

16. Ebert MS, Neilson JR and Sharp PA: MicroRNA sponges: Competitive inhibitors of small RNAs in mammalian cells. Nat Methods 4: 721-726, 2007.

17. Kloosterman WP, Lagendijk AK, Ketting RF, Moulton JD and Plasterk RH: Targeted inhibition of miRNA maturation with morpholinos reveals a role for miR-375 in pancreatic islet development. PLoS Biol 5: e203, 2007.

18. Stenvang J, Petri A, Lindow M, Obad S and Kauppinen S: Inhibition of microRNA function by antimiR oligonucleotides. Silence 3: 1, 2012.

19. Wang GJ, Liu GH, Ye YW, Fu Y and Zhang XF: The role of microRNA-1274a in the tumorigenesis of gastric cancer: Accelerating cancer cell proliferation and migration via directly targeting FOXO4. Biochem Biophys Res Commun 459: 629-635, 2015.
20. Sand M, Skrygan M, Sand D, Georgas D, Gambichler T, Hahn SA, Altmeyer P and Bechara FG: Comparative microarray analysis of microRNA expression profiles in primary cutaneous malignant melanoma, cutaneous malignant melanoma metastases, and benign melanocytic nevi. Cell Tissue Res 351: 85-98, 2013.

21. Zhou C, Liu J, Li Y, Liu L, Zhang X, Ma CY, Hua SC, Yang M and Yuan Q: microRNA-1274a, a modulator of sorafenib induced a disintegrin and metalloproteinase 9 (ADAM9) down-regulation in hepatocellular carcinoma. FEBS Lett 585: 1828-1834, 2011.

22. Yan H, Wang S, Yu H, Zhu J and Chen C: Molecular pathways and functional analysis of miRNA expression associated with paclitaxel-induced apoptosis in hepatocellular carcinoma cells. Pharmacology 92: 167-174, 2013.

23. Ezzie ME, Crawford M, Cho JH, Orellana R, Zhang S, Gelinas R, Batte K, Yu L, Nuovo G, Galas D, et al: Gene expression networks in COPD: microRNA and mRNA regulation. Thorax 67: 122-131, 2012.

24. Yoshino H, Enokida H, Itesako T, Tatarano S, Kinoshita T, Fuse M, Kojima S, Nakagawa M and Seki N: Epithelialmesenchymal transition-related microRNA-200s regulate molecular targets and pathways in renal cell carcinoma. J Hum Genet 58: 508-516, 2013.

25. García de Vinuesa A, Abdelilah-Seyfried S, Knaus P, Zwijsen A and Bailly S: BMP signaling in vascular biology and dysfunction. Cytokine Growth Factor Rev 27: 65-79, 2016

26. Heldin $\mathrm{CH}$, Miyazono K and ten Dijke P: TGF-beta signalling from cell membrane to nucleus through SMAD proteins. Nature 390: 465-471, 1997.

27. Jang CW, Chen CH, Chen CC, Chen JY, Su YH and Chen RH: TGF-beta induces apoptosis through Smad-mediated expression of DAP-kinase. Nat Cell Biol 4: 51-58, 2002.

28. Halvorsen AR, Kristensen G, Embleton A, Adusei C, BarretinaGinesta MP, Beale P and Helland A: Evaluation of prognostic and predictive significance of circulating MicroRNAs in ovarian cancer patients. Dis Markers 2017: 3098542, 2017. doi. org/10.1155/2017/3098542.

29. Slattery ML, Trivellas A, Pellatt AJ, Mullany LE, Stevens JR, Wolff RK and Herrick JS: Genetic variants in the TGF $\beta$-signaling pathway influence expression of miRNAs in colon and rectal normal mucosa and tumor tissue. Oncotarget 8: 16765-16783, 2017.

30. Allison SE, Chen Y, Petrovic N, Zimmermann S, Moosmann B, Jansch M, Cui PH, Dunstan CR, Mackenzie PI and Murray M: Activation of the pro-migratory bone morphogenetic protein receptor 1B gene in human MDA-MB-468 triple-negative breast cancer cells that over-express CYP2J2. Int J Biochem 80: 173-178, 2017. 